\title{
Determination of dominant type of defects in Cz-Si single crystals after irradiation with high-energy electrons
}

\author{
V.V. Dovganyuk ${ }^{1}$, I.M. Fodchuk ${ }^{1,2}$, O.G. Gimchinsky ${ }^{1}$, A.V. Oleinych-Lysyuk ${ }^{1}$, A.I. Nizkova ${ }^{2}$ \\ ${ }^{l}$ Yu. Fedkovich Chernivtsi National University, 2, Kotsyubinsky str., 58012 Chernivtsi, Ukraine \\ ${ }^{2}$ G.V. Kurdyumov Institute for Metal Physics, NAS of Ukraine, Kyiv
}

\begin{abstract}
Theoretical and experimental studies of silicon crystals irradiated with highenergy electrons $(E=18 \mathrm{MeV})$ were made using the method of full integral X-ray reflectivity. To explain peculiarities of full integral reflectivity behaviour versus radiation dose and the order of reflection, the relationships of generalized dynamic theory of the Bragg X-ray diffraction in crystal comprising several defect types were used. The presence of several types of dominant defects in crystals allows closer tracing the dynamics of full integral reflectivity change versus the order of reflection and radiation dose, as well as estimating the contribution of each defect type to the diffusion component of X-ray scattering.
\end{abstract}

Keywords: irradiation with high-energy electrons, X-ray reflectivity, Cz-Si, oxygencontaining defects.

Manuscript received 11.03.06; accepted for publication 29.03.06.

\section{Introduction}

Structural changes in Czochralski grown silicon $(\mathrm{Cz}-\mathrm{Si})$ single crystals in the course of natural ageing and after high-energy irradiation with particles were studied in a series of papers $[1,7,9,10]$. After high-energy irradiation with particles and formation of radiation defects, large concentrations of various-size microdefects [2-5], meta- and bistable radiation defects and their systems with oxygen-containing defects $[6,9]$ appear at the initial ageing stages. This is also confirmed by the data obtained from the thickness distribution of the X-ray intensity in acoustically excited silicon crystals $[6,9]$.

In the course of natural ageing of electronirradiated crystals, alongside with formation of secondary radiation defects, the authors of $[1,7,10]$ observed enlargement of intrinsic microdefects and formation of essential internal stresses $\left(\varepsilon \sim 2 \cdot 10^{-5}\right)$ in $\mathrm{Si}$. Two basic stages of defect formation were determined: 1) intensive enlargement of existing defects; 2) formation of small $\mathrm{SiO}_{\mathrm{x}}$ microdefects against increased concentration of secondary radiation defect systems. However, in these papers no mention has been made of the type and quantitative changes in microdefect sizes depending on the radiation dose.

Silicon irradiation with various doses of highenergy electrons leads to activation of oxidation processes [11]. In the bulk of silicon crystal, there is intensive generation of new cluster formations and disintegration of growth cluster formations that are $\mathrm{SiO}_{2}$ precipitates by assumption of the authors [12]. Due to bulk changes in the region of precipitate formation, the Franck dislocation loop can originate due to oxygen diffusion from a matrix to crystal surface. Besides, such processes, as a rule, are accompanied by formation of packing defects that are an origination of silicon-oxygen clusters in the planes (111) [13]. Oxygen atoms in such clusters are implanted into interstitial locations between the pairs of silicon atoms located along the directions [111] causing a compressive strain of matrix outside silicon crystal. For further growth of oxygen-containing clusters the interstitial atoms should be implanted into surrounding lattice. When the concentration of interstitial atoms in such regions reaches a certain critical value, their condensation with the cluster formation surrounded by a dislocation loop is possible. Such cluster formations have smaller effective sizes than the dislocation loops.

\section{Formulation of the problem and object of investigation}

The purpose of this paper is to determine the dominant type of defects in silicon crystals depending on highenergy electron irradiation dose being based on the method of full integral X-ray reflectivity. 
Dislocation-free $\mathrm{Cz}-\mathrm{Si}$ single crystals doped with boron ions that comprise the horizontal and radial growth bands with the period $\sim 50 . .200 \mu \mathrm{m}$ as well as the microdefects of various type were chosen as the objects of investigation. According to the data of IR spectroscopy, the oxygen concentration therein is $n \sim 10^{18} \mathrm{~cm}^{-3}$ and concentration of boron ions is $n \sim 10^{16} \mathrm{~cm}^{-3}$.

Two series of samples were prepared for investigation (Table) that differed in thickness and were produced from the same original crystal. Each of the series comprised three crystals. One of the crystals in series was chosen as a test sample. The electron irradiation energy $E=18 \mathrm{MeV}$, radiation dose $D$ for each sample was 1.8, 3.6, 2.7, $5.4 \mathrm{kGray}$, respectively (in Table the specific radiation dose is indicated based on $1 \mathrm{~mm}$ of crystal thickness). To remove the damaged surface layer before and after irradiation, all the samples were processed with standard chemical and mechanical methods (for the depth to $400 \mu \mathrm{m}$ ).

Table.

\begin{tabular}{|c|c|c|c|}
\hline $\begin{array}{c}\text { Sample } \\
\text { number }\end{array}$ & $\begin{array}{c}\text { Radiation dose, } \\
\text { kGray }\end{array}$ & $\begin{array}{c}\text { Thickness, } \\
\mathrm{mm}\end{array}$ & $\begin{array}{c}\text { Specific } \\
\text { radiation dose, } \\
\text { Gray/mm }\end{array}$ \\
\hline 1 & Test & 4.271 & Test \\
\hline 1a & 1.8 & 4.263 & 421 \\
\hline 1b & 3.6 & 4.261 & 844 \\
\hline 2 & Test & 10.27 & Test \\
\hline 2a & 2.7 & 10.264 & 262 \\
\hline $2 \mathrm{~b}$ & 5.4 & 10.267 & 525 \\
\hline
\end{tabular}

\section{Experimental investigations}

The structural changes caused by irradiation with high-energy electrons were studied by the full integral reflectivity method. Investigations were performed on a two-crystal diffractometer in the Bragg diffraction geometry for (111), (333), (444), (555) reflections of Mo $K_{\alpha}$-radiation. In the measurements, the symmetric reflection (220) from Si crystal monochromator was used. Selection of various diffraction orders allowed tracing the dynamics of changes in the concentration and microdefect size along the crystal thickness.

Experimental dependences of the full integral reflectivity for X-rays normalized with respect to perfect sample on the order of reflection (extinction thickness $\Lambda_{h k l}$ ) is shown in Fig. 1. These dependences are not monotonic. As compared to the test sample, the full integral reflectivity of irradiated crystals is increased, with the maximum gain in reflectivity observed at the radiation dose $1.8 \mathrm{kGray}$ and the minimum one at 3.6 kGray.

For the test samples 1 and 2, in the dependences $\rho\left(\Lambda_{h k l}\right)$ (Fig. 1) one can see a similar character of gain in the full integral reflectivity, but there are somewhat different value of bend of curves $\rho^{1}\left(\Lambda_{h k l}\right)$ and $\rho^{2}\left(\Lambda_{h k l}\right)$ in the interval between $\Lambda_{333}$ and $\Lambda_{444}$ extinction thicknesses and reduction of $\rho^{2}\left(\Lambda_{h k l}\right)$ in the interval between $\Lambda_{444}$ and $\Lambda_{555}$. It testifies to the fact that the test samples are somewhat different in defect structure.

At the same time, for the irradiated samples of series under study we have essentially different dependences $\rho\left(\Lambda_{h k l}\right)$ both in the character and the values. It is particularly pronounced for the crystal 1a (Fig. 1a). Here, the dependence $\rho^{1 \mathrm{a}}\left(\Lambda_{h k l}\right)$ is almost constant, but its value is much in excess of $\rho^{1}\left(\Lambda_{h k l}\right)$ of the test sample.

The curve $\rho^{1 \mathrm{~b}}\left(\Lambda_{h k l}\right)$ for the thicknesses up to $\Lambda_{444}$ has the same behaviour as $\rho^{1}\left(\Lambda_{h k l}\right)$ and is located below it, and its values, on the average, are almost half the value $\rho^{1 \mathrm{a}}\left(\Lambda_{h k l}\right)$. The dependences $\rho\left(\Lambda_{h k l}\right)$ in Fig. $1 \mathrm{~b}$ for the samples of series 2 look somewhat different. Bends or slight inflections are observed in the dependences $\rho^{2, \mathrm{a}, \mathrm{b}}\left(\Lambda_{h k l}\right)$ in the range of 23 to $60 \mu \mathrm{m}$. It can be related to the presence of an increased content of defects at this depth - growth of the band [11]. The curves $\rho^{2 \mathrm{a}}\left(\Lambda_{h k l}\right)$ and $\rho^{2 \mathrm{~b}}\left(\Lambda_{h k l}\right)$ are lower than the $\rho^{2}\left(\Lambda_{h k l}\right)$ one, monotonically growing with increasing the order of reflection.

\section{Theoretical part}

To explain the peculiarities in behaviour of the full integral reflectivity versus the radiation dose in Fig. 1, let us use the generalized dynamic theory of the Bragg diffraction of X-rays in crystal comprising defects of several types [14-22].

We have considered several possible combinations of the presence of microdefects with different sizes and concentrations in crystals under study. The best agreement between experimental and calculated values of the full integral reflectivity of X-rays has been obtained for models comprising the following dominant defect types:

1) disc-like clusters, small spherical clusters $-\mathrm{SiO}_{2}$ precipitates, dislocation loops;

2) disc-like clusters $-\mathrm{SiO}_{2}$ precipitates, dislocation loops.

In an arbitrary case of the Bragg diffraction according to $[14,15]$, a relationship describing the full integral reflectivity of X-rays for single crystal, comprising damaged near-surface layer of thickness $t_{k s l}$, statistically distributed various types of microdefects as well as combining boundary cases of thin $\left(\mu_{0} t<<1\right)$ and thick $\left(\mu_{0} t>>1\right)$ crystals, is of the form: 

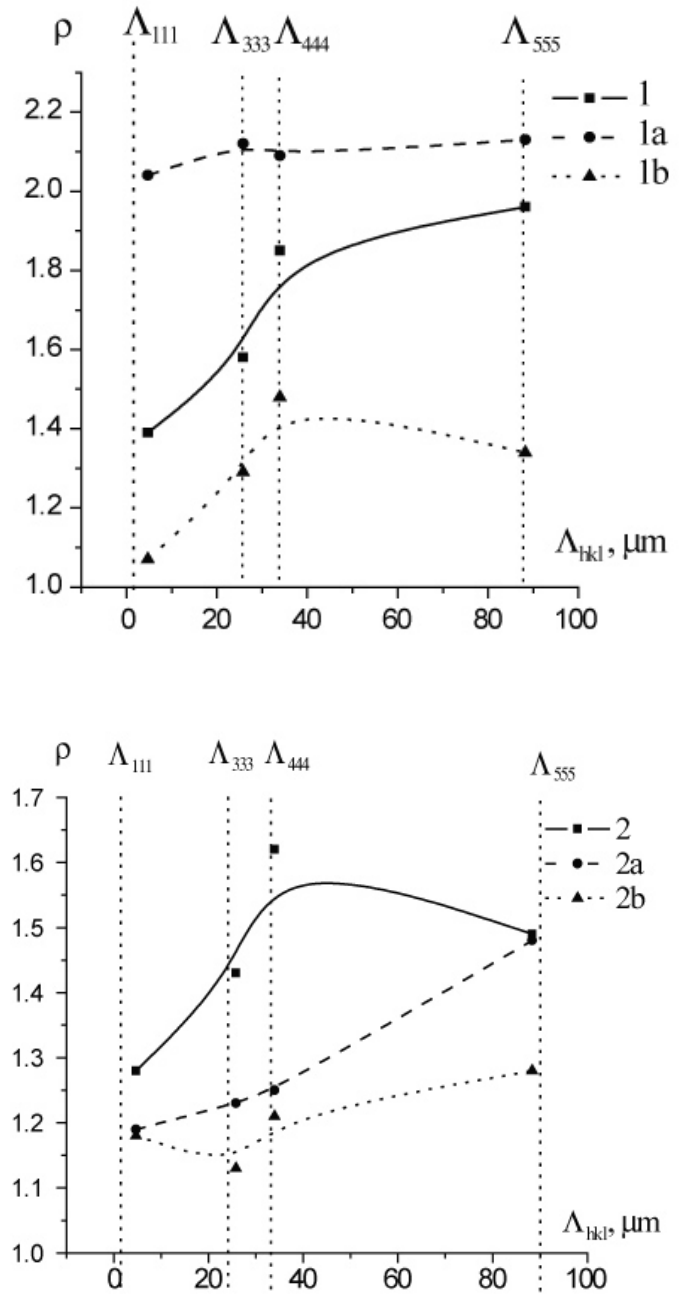

Fig. 1. Dependences of the normalized full integral reflectivity ( $\rho)$ on the order of reflection (the values of extinction thicknesses) of sample series 1 (a) and 2 (b). (The sample numbers are indicated in the inserts, the specific radiation dose is indicated in Table; $\rho_{\text {exp }} \approx \rho_{\text {theor }}=\rho$.)

$R_{i}=\left(R_{i}^{S D D}+R_{i k s c}\right) \times \exp \left[-\mu_{0} t_{k s l}\left(1 / \gamma_{0}+1 /\left|\gamma_{H}\right|\right)\right]$,

where $R_{i k s c}=C^{2}\left(Q / \gamma_{0}\right) t_{k s l}=C^{2}\left(Q / \gamma_{0}\right) k \Lambda(a / d)$,

$R_{i}^{S D D}=R_{i}^{\mathrm{dyn}} \cdot P \cdot E+R_{i P} \cdot \Pi\left(1-E^{2}\right)$

where $R_{i}^{\mathrm{dyn}}=(16 / 3 \pi) C Q \Lambda / \gamma_{0} ; \quad R_{i P}=C^{2} Q t / \gamma_{0}$ is the full integral reflectivity of a perfect crystal [14];

$\Pi\left(\mu^{*}, t\right) \cong\left\{\begin{array}{l}\left(2\left(\mu_{0}+\mu^{*}\right) t / \gamma\right)^{-1}, \quad \text { at } \mu_{0} t>>1, \\ \left(1+\left(\mu_{0}+\mu^{*}\right) t / \gamma\right)^{-1}, \text { at } \mu_{0} t<1, t>>\Lambda,\end{array}\right.$

here $1 / \gamma=\left(1 /\left|\gamma_{0}\right|+1 /\left|\gamma_{H}\right|\right) / 2 ; \quad \Lambda=\lambda\left(\gamma_{0} \gamma_{H}\right)^{1 / 2} /\left(2 \pi C\left|\chi_{H r}\right|\right)=$ $=\Lambda / 2 \pi, \Lambda$ is the extinction thickness, $P \cong 1-3 \pi s / 4$ at $s<<1 ; s=\left(\mu_{0}+\mu_{d s}^{0}\right) \Lambda E / \gamma C ; C=1$ or $\cos \left(2 \theta_{\mathrm{B}}\right)$ is the polarization factor, $Q=\left(\pi\left|\chi_{H r}\right|^{2}\right) /\left[\lambda \sin \left(2 \theta_{\mathrm{B}}\right)\right]$ is the reflectivity per unit path length, $\chi_{H r}$ is the real part of the
Fourier component polarizability, $t$ is the crystal thickness, $\quad \gamma_{0}=\cos \theta_{\mathrm{B}} \cdot \cos \psi \cdot \sin \alpha+\sin \psi \cdot \sin \theta_{\mathrm{B}}, \quad \gamma_{H}=$ $=\cos \theta_{\mathrm{B}} \cdot \cos \psi \cdot \cos \alpha-\cos \psi \cdot \sin \theta_{\mathrm{B}}$ are the guiding cones of wave vectors for the flat wave incident on crystal with respect to the internal normal to input crystal surface and the diffracted wave, respectively. In a case of the symmetric geometry of the Bragg diffraction $\gamma_{0}=\gamma_{H}=\sin \theta_{\mathrm{B}}, \quad \mu_{0}$ is the linear coefficient of photoelectric absorption.

For a case of homogeneous distribution of the defects with the radius $R_{0}$ and concentration $c$, the following expression holds [17-19]:

$\mu_{d s}^{0}=c E^{2} C^{2} m_{0} B ; m_{0}=\pi v_{c} H^{2}\left|\chi_{H r}\right|^{2} /\left(2 \lambda^{2}\right)$,

$B=b_{1}+b_{2} \ln \left(e / r_{0}^{2}\right), b_{1}=B_{1}+B_{2} / 3$,

$b_{2}=B_{1}+\cos ^{2} \theta_{\mathrm{B}} \cdot B_{2} / 2$,

where $v_{c}$ is the volume of unit cell, $r_{0}=R_{0} / \Lambda, H$ is the diffraction vector module, $e$ is the natural logarithm base, and it is expected that $r_{0}<1$.

For spherical clusters of the radius $R_{0}[18,21]$ : $B_{1}=0, \quad B_{2}=\left(4 \pi A_{\mathrm{cl}} / v_{c}\right)^{2}, \quad A_{\mathrm{cl}}=\Gamma \varepsilon R_{0}^{3}$ is the cluster power, $\varepsilon$ is the relative deformation on the cluster boundary $\Gamma=(1+v) /[3(1-v)]$.

For disc-like clusters of the radius $R_{0}$ and thickness $h_{p} \quad[20,22]: \quad B_{1}=B_{2}=\left(4 \pi A_{\mathrm{cl}} / v_{c}\right)^{2}$, $A_{\mathrm{cl}}=\Gamma \varepsilon R_{0}^{2} h_{p} / 2$ is the power of a disc-like cluster.

For randomly distributed dislocation loops: $B_{1}=4\left(\pi b R_{0}^{2} / v_{c}\right)^{2} / 15, B_{2}=\beta \cdot B_{1}, \beta=\frac{\left(3 v^{2}+6 v+1\right)}{4\left(1-v^{2}\right)}, v$ is the Poisson ratio, $b$ is the Burgers vector module.

For $\mu_{d s}^{0}<<\mu_{0}$ i $r_{0}<<1$, the approximate relationship holds:

$\mu^{*} \approx \mu_{d s}^{0} f_{\mu}\left(r_{0}\right)$,

where $f_{\mu}\left(r_{0}\right)=\left(\frac{5+2 r_{0} \ln r_{0}-(3 / 8) r_{0}}{3\left(1-\ln r_{0}\right)}\right)-$ for dislocation loops, $f_{\mu}\left(r_{0}\right)=\left(\frac{4+r_{0} \ln r_{0}-2 r_{0}}{5-6 \ln r_{0}}\right)-$ for clusters.

The static Debye-Waller factor $L_{H}=-\ln E$ is related to the characteristics of defects as follows [15, 21, 22]:

$L_{H} \approx 0.5 c v_{c}^{-1} R_{0}^{3}(H \cdot b)^{3 / 2}$

- for the dislocation loops,

$L_{H} \approx 0.5 c n_{0} \eta^{2}\left(1-\eta^{2} / 100\right)$

- for the clusters $\left(\eta^{2}<<10\right)$,

$L_{H} \approx c n_{0} \eta^{3 / 2}$

- for clusters $\left(\eta^{2}>>10\right)$. 
Here $n_{0}$ is the number of matrix unit cell substituted by the cluster: $n_{0}=(4 / 3) \pi R_{0}^{3} / v_{c}-$ for spherical clusters and $n_{0}=\pi R_{0}^{2} h_{p} / v_{c}$ - for disc-like clusters; $\eta=\alpha_{0} n_{0}^{1 / 3} h$, $\alpha_{0}=\Gamma \varepsilon\left(6 \pi^{2} / v_{0}\right)^{1 / 3}, v_{0}$ is the number of atoms in matrix cubic cell; $h=H a / 2 \pi, a$ is the lattice parameter.

Note that this theory is characterized in that the value of the full integral reflectivity $R_{i}$ of crystal is related to the basic characteristics of defects $\left(c, R_{0}, \varepsilon, b\right)$ through the parameters $E, \mu_{d s}^{0}$ and $\mu^{*}$.

\section{Analysis of investigation results}

Based on the relationship (1) with regard to (3)-(7), the theoretic dependences $\rho_{\text {theor }}$ (of normalized full integral reflectivity) were matched to the experimental dose. The best agreement between the experimental and theoretical dependences of the full integral reflectivity (in Fig. 1 $\rho=\rho_{\text {exp }} \approx \rho_{\text {theor }}$, the deviation does not exceed $1 \%$ ) was obtained for the first model - three types of dominant defects in crystal. It allowed to determine the characteristics of dominant defects (concentration and dimensions) in crystals before and after irradiation (Figs 2-4).

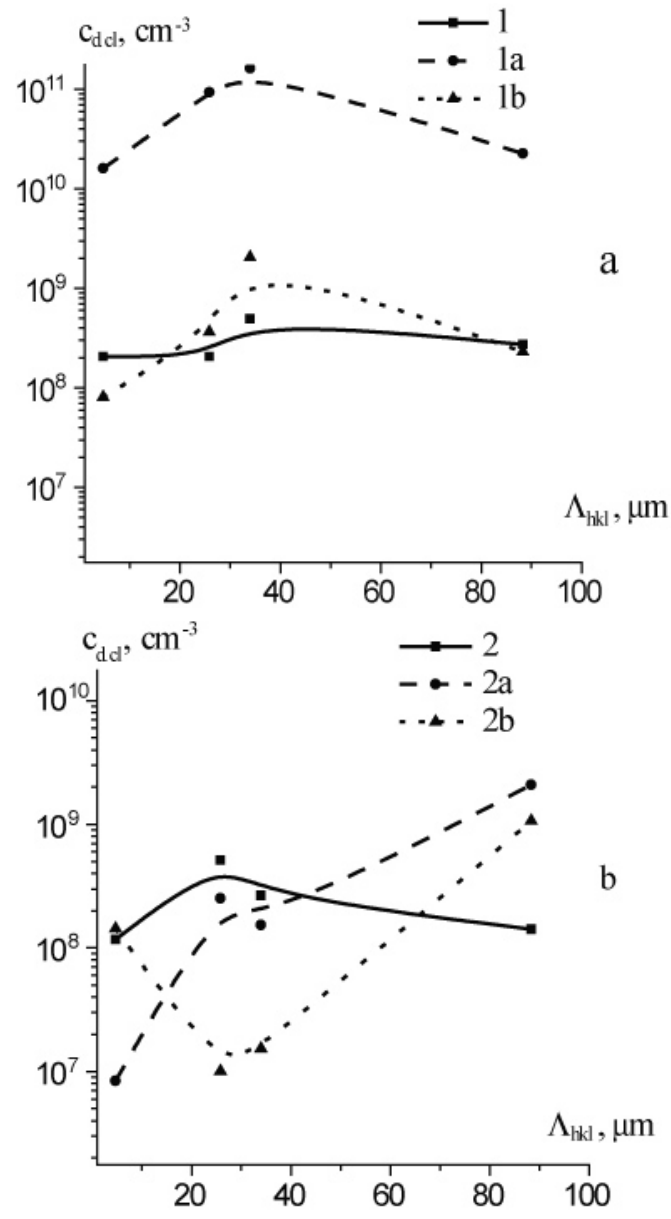

Fig. 2. Dependences of the concentration of disc-like clusters on the order of reflection for the sample series 1 (a) and 2 (b) $\left(R_{\text {d.cl }}=0.65 \mu \mathrm{m}\right)$.
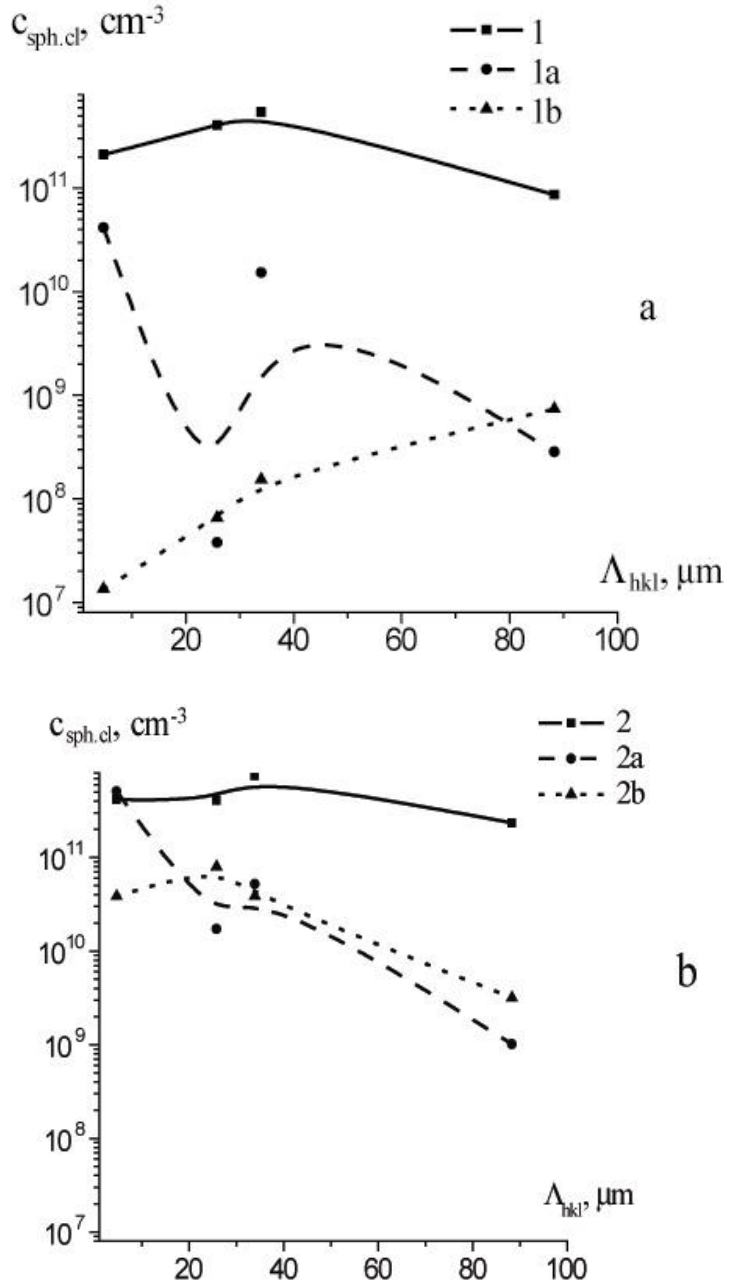

Fig. 3. Dependences of the concentration of spherical clusters on the order of reflection for the sample series 1 (a) and 2 (b) $\left(R_{\text {sph.cl }}=8 \mathrm{~nm}\right)$.

For the test samples, no essential changes are observed in the concentration dependences of dominant defects versus the order of reflection $c\left(\Lambda_{h k l}\right)$ (Figs 2 to 4 ). Note that the chosen dimensions $\left(R_{\mathrm{d} . \mathrm{cl}}=0.65 \mu \mathrm{m}, R_{\mathrm{sph} . \mathrm{cl}}=\right.$ $\left.8 \mathrm{~nm}, R_{L}=1.8 \mu \mathrm{m}\right)$ of defects are based on the reported data $[1,7,9,10,23]$ and results given in Figs 5 to 8 .

For the irradiated samples $1 \mathrm{a}$ and $1 \mathrm{~b}$ in the dependences $c\left(\Lambda_{h k l}\right)$ (Figs 2a-4a) there is a number of specific features. In particular, for the sample 1a there is a considerable increase in the concentration of disc-like clusters (Fig. 2a) and dislocation loops (Fig. 4a) with respect to the test sample. Concentration of spherical clusters with increasing the order of reflection is decreased (Fig. 3a).

For the irradiated samples of series 2 (Figs $2 b-4 b$ ) the concentration dependences of dominant defects versus the order of reflection are more complicated than those for the test sample. For dislocation loops, the concentration curves $c_{L}^{2 \mathrm{a}}\left(\Lambda_{h k l}\right)$ and $c_{L}^{2 \mathrm{~b}}\left(\Lambda_{h k l}\right)$ (Fig. $4 \mathrm{~b}$ ) have higher values (more than an order) than the dependence $c_{L}^{2}\left(\Lambda_{h k l}\right)$ of the test sample. 

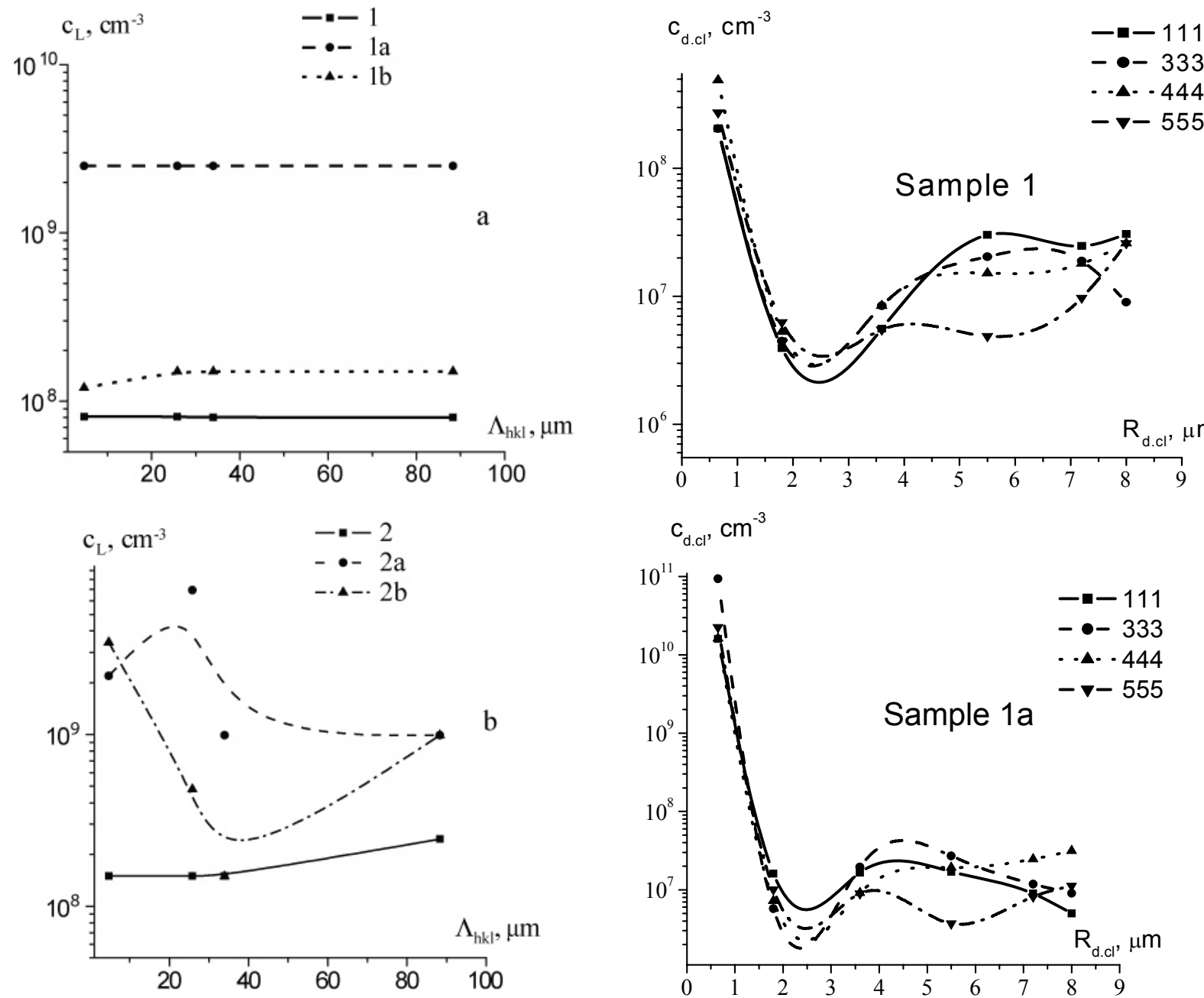

Fig. 4. Dependences of the concentration of dislocation loops on the order of reflection for the sample series 1 (a) and 2 (b) $\left(R_{L}=1.8 \mu \mathrm{m}\right)$

Along with this, in Fig. $2 b$ for reflection (111), the concentration of disc-like clusters for the sample $2 \mathrm{a}$ is much less (by an order) than in the original one $c_{\mathrm{d} . \mathrm{cl}}^{2 \mathrm{a}}\left(\Lambda_{111}\right)<c_{\mathrm{d} . \mathrm{l} l}^{2}\left(\Lambda_{111}\right)$. With increasing the order of reflection, $c_{\mathrm{d} . \mathrm{cl}}^{2 \mathrm{a}}\left(\Lambda_{h k l}\right)$ is increased and crosses $c_{\mathrm{d} . \mathrm{cl}}^{2}\left(\Lambda_{h k l}\right)$ at (444) reflection order. For the sample $2 \mathrm{~b}$ at first $c_{\mathrm{d} . \mathrm{cl}}^{2 \mathrm{~b}}\left(\Lambda_{111}\right) \approx c_{\mathrm{d} . \mathrm{cl}}^{2}\left(\Lambda_{111}\right)$, then it is decreased and reaches the minimum value $\left(c_{\mathrm{d} . \mathrm{ll}}^{2 \mathrm{~b}}\left(\Lambda_{333}\right) \approx 10^{7} \mathrm{~cm}^{-3}\right)$, then it is increased and crosses $c_{\text {d.cl }}^{2}\left(\Lambda_{h k l}\right)$ at $\Lambda=70 \mu \mathrm{m}$.

Worthy of special mention is behaviour of the concentration dependences $c_{\text {sph.cl }}\left(\Lambda_{h k l}\right)$ of small spherical clusters versus the order of reflection (Fig. 3a, b). If for the sample 1a, with increasing the order of reflection, the concentration of disc-like clusters $c_{\text {d.cl }}$ is increased (Fig. 2a), the concentration of small spherical clusters $c_{\text {sph.cl }}$ is decreased (Fig. 3a). tration values $c_{\text {sph.cl }}^{2 \mathrm{a}, 2 \mathrm{~b}}$ approach to $c_{\text {sph.cl }}^{2}$ of the test sample (Fig. 3b). 

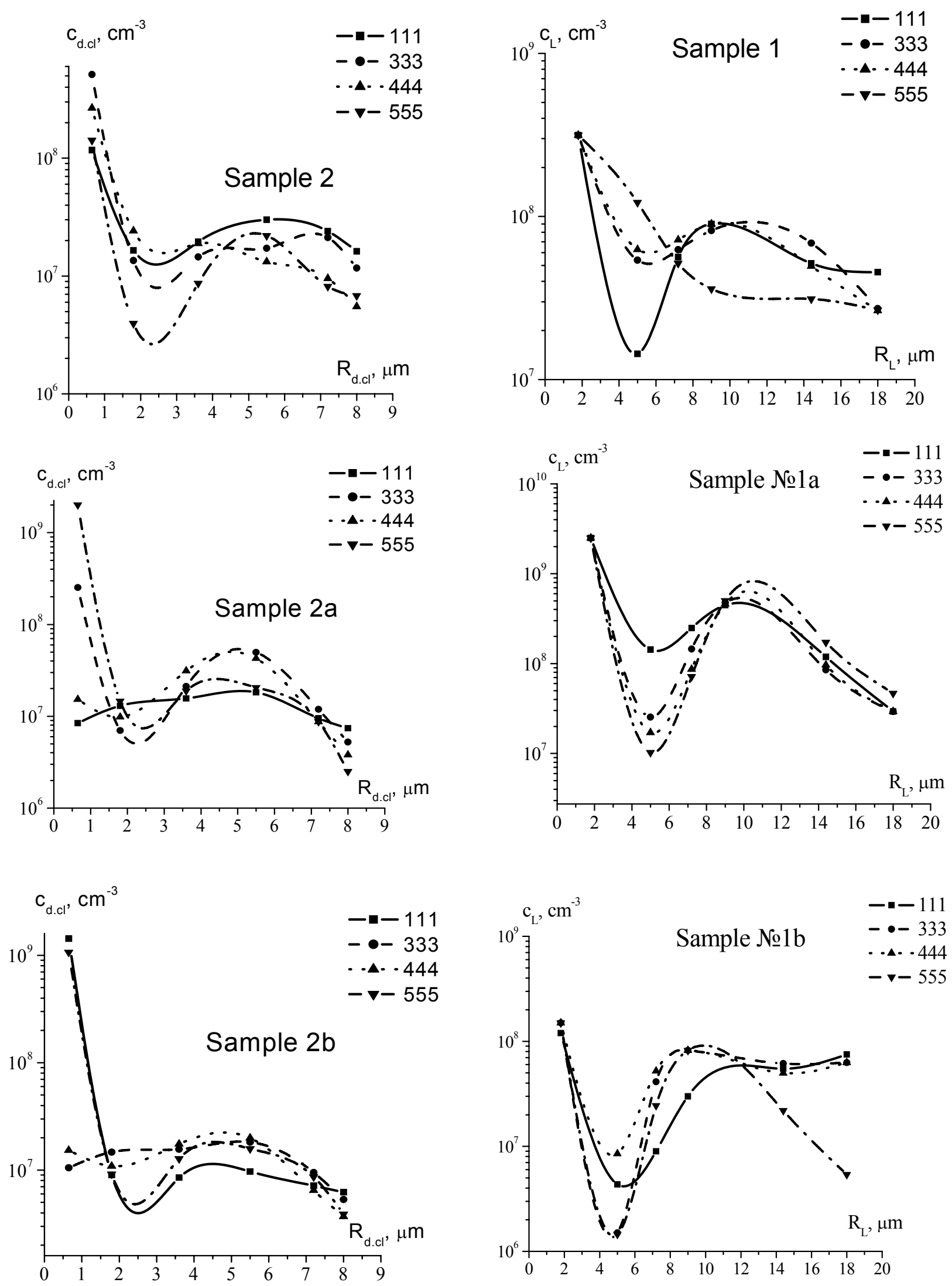

Fig. 6. Concentration-size profiles of disc-like clusters of the sample series 2 .

Fig. 7. Concentration-size profiles of dislocation loops for the sample series 1 .

(C) 2006, V. Lashkaryov Institute of Semiconductor Physics, National Academy of Sciences of Ukraine 

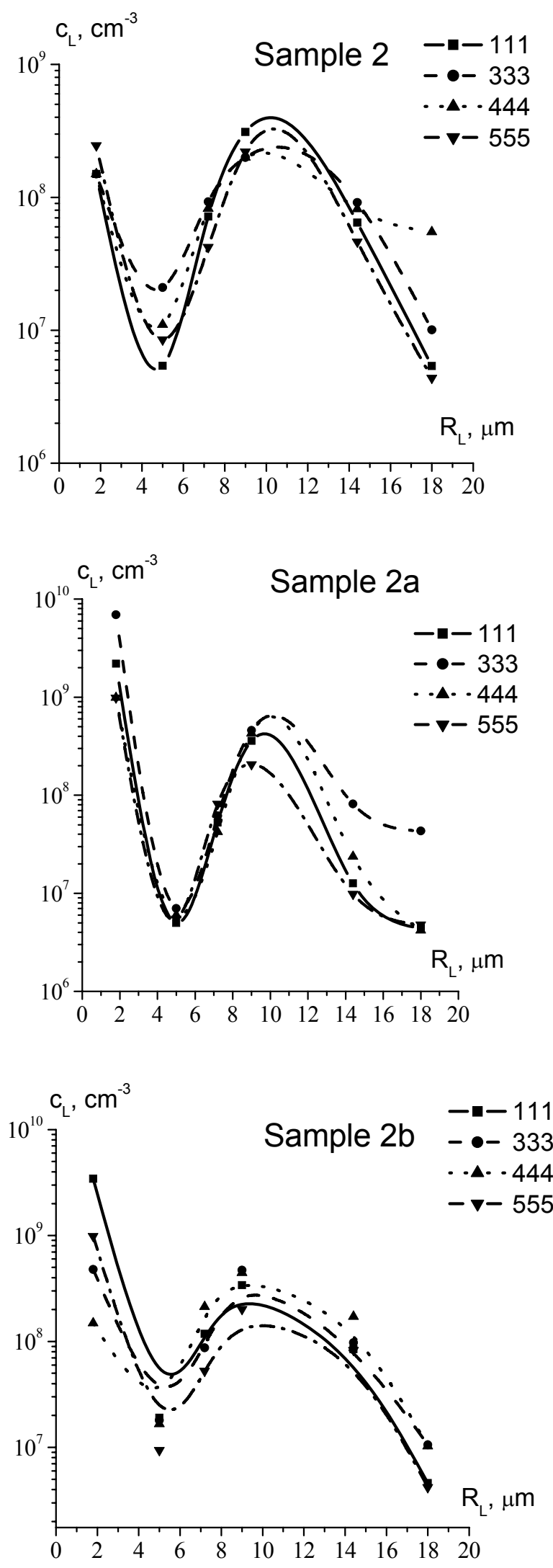

Fig. 8. Concentration-size profiles of dislocation loops of the sample series 2 .
The results obtained allow to assume that the small spherical clusters after irradiation are sinks for vacancies or interstitial atoms, and on achieving the certain critical dimensions, they can be transformed into disc-like clusters and dislocation loops [2-5, 8, 12-14].

Note that the concentration dependences $c_{\text {d.cl }}\left(\Lambda_{h k l}\right)$

(Fig. 2) to a certain degree account for behaviour of the full integral reflectivity in Fig. 1, as long as these defects make the greatest contribution to the value of full integral reflectivity.

The results shown in Figs 2-4 do not completely describe the dynamics of change in the concentration of dominant defects in crystals versus the radiation dose and the order of reflection, hence it is necessary to study the dynamics of concentration-size dependences of dominant defects before and after irradiation $[7,8,10]$.

Using the relationship between the concentration $c$ and defect size $R_{0}$, through structural parameters $L_{H}$ and $\mu_{d s}$ (relationships (3)-(7)), the concentration-size dependences for disc-like clusters and dislocation loops for various orders of reflection are calculated in Figs 5-8. All the dependences have been averaged by the least-squares method.

In the dependences $c_{\text {d.cl }}(R)$ (Figs 5,6$)$ for the test samples 1 and 2, a typical inflection in the range of values $R_{\text {d.cl }}=3.6 \ldots 7.2 \mu \mathrm{m}$ is observed with further increase of the dependence $c_{\mathrm{d} . \mathrm{cl}}^{1}(R)$ and "saturation" of the dependence $c_{\text {d.cl }}^{2}(R)$. For the irradiated samples of series 2 , the dependences $c_{\mathrm{d} . \mathrm{cl}}^{2 \mathrm{a}, 2 \mathrm{~b}}(R)$ have a complicated character of changes, certain peculiarities of behaviour versus the order of reflection are observed: oscillatingdecreasing behaviour $c_{\mathrm{d} . \mathrm{cl}}^{2 \mathrm{a}}(R)$ for reflections (111) and (333) and similar behaviour $c_{\mathrm{d} . \mathrm{cl}}^{2 \mathrm{~b}}(R)$ for reflections (333) and (444). It can be related to the fact that highenergy electron irradiations stimulated the processes of ordering (relaxation) of the defect structure of the samples.

If the concentration values of dependences $c_{\mathrm{d} . \mathrm{ll}}^{1 \mathrm{~b}}(R), \quad c_{\mathrm{d} . \mathrm{cl}}^{2 \mathrm{a}}(R), \quad c_{\mathrm{d} . \mathrm{cl}}^{2 \mathrm{~b}}(R)$ are changed in the same interval - from $5 \cdot 10^{6}$ to $10^{9} \mathrm{~cm}^{-3}$, for $c_{\mathrm{d} . \mathrm{cl}}^{\text {la }}(R)$ the interval of changes is much broader $-5 \cdot 10^{6} \ldots 10^{11} \mathrm{~cm}^{-3}$. As this takes place, the sample la has the highest content of small-size disc-like clusters. It can testify to the intensive process of size reduction of A-type microdefects and appearance of new cluster sources after high-energy electron irradiation. This assumption is also confirmed by considerable gain in the full integral reflectivity of the sample la as compared to the test sample.

The concentration-size profiles $c_{L}(R)$ for dislocation loops in Figs 7 and 8 are similar for all the samples of series 2 with typical inflections in the range of close values of $R_{L}$. 
A somewhat different situation is observed for irradiated samples of series 1: with the large $R_{L}$ values the concentration of dislocation loops for sample $1 \mathrm{a}$ is decreased, whereas for the test sample and sample $1 \mathrm{~b}$ it is changed only slightly (Fig. 7). Besides, the dependences $c_{L}^{\text {la }}(R)$ are characterized by an order of magnitude greater values of concentrations for small dislocation loops. A similar situation holds for the dependence $c_{\mathrm{d} . c \mathrm{l}}^{\text {la }}(R)$. Note somewhat complicated character of the dependence $c_{L}^{1}(R)$, in particular, its monotonic decrease for (555) reflection. It also allows assuming the availability in studying the crystals of the increased defect content (growth bands) at the depths of order 23-60 $\mu \mathrm{m}$.

The obtained results, in general, are corroborated by calculations in a case of two types of dominant defects presented in crystal - disc-like clusters and dislocation loops (the second model of defect structure). However, this model does not provide a satisfactory agreement between the calculated and experimental values of the full integral reflectivity, besides, the X-ray scattering in damaged near-surface layer contributes considerably to the value of full integral reflectivity $(\sim 20-25 \%)$, whereas its contribution to full integral reflectivity, according to the first model, is less than 5-10\%.

All the above mentioned peculiarities in dynamics of changes of the concentration-size dependences for dominant defects in crystals under study versus the order of reflection and radiation dose are attributable to the following reasons. As is known, $\mathrm{Cz}-\mathrm{Si}$ single crystals are exposed to heat treatment $[23,24]$, in addition to growth the microdefects comprise oxygen aggregates originating at low-temperature heat treatment. These low-temperature oxygen aggregates due to thermal shock (irradiation with high-energy particles, annealing) can form silicon precipitates which due to emission of vacancies or interstitial atoms will nucleate as clusters or stimulate reconstruction of the growth clusters of interstitial type into $\mathrm{SiO}_{x}$ precipitates. Due to condensation of intrinsic interstitial silicon atoms and reduction of stress field around precipitates which, respectively, will be miniaturized, the new dislocation loops will be generated or those available in crystal will be reduced in size. This process can be accompanied by formation of smaller-size microdefects of B-type [11-13].

With high-temperature treatment or high-energy irradiation with large doses of electrons, the $\mathrm{SiO}_{\mathrm{x}}$ precipitates together with the growth defects (dislocation loops, point defect clusters) serve as sinks for atoms of interstitial silicon, which will stimulate enlargement of growth A-defects (clusters) or their size reduction and, respectively, nucleation of new clusters. To a certain extent this reasoning agrees with the data reported in [11-13].

Size reduction of disc-like clusters and dislocation loops along with their concentration growth, brings about enlargement of the Debye-Waller factor $L_{H}$ (Eqs (5)-(7)) and the diffusion scattering factor $\mu_{d s}$ (3) that entails a gain in the full integral reflectivity value. This can be accounted for degradation of structural perfection degree of the sample 1a as compared to the test one. For other irradiated samples, no essential changes in the size of disc-like clusters and dislocation loops as compared to the test ones are observed. It is attributable to two equivalent processes - size reduction of growth Adefects and parallel nucleation of B-microdefects of approximately the same size as A-microdefects of the reduced size. This assumption is argued by the almost constant dependences $c_{\mathrm{d} . \mathrm{cl}}^{2 \mathrm{a}}(R)$ for reflectivity (111) and (333) as well as $c_{\mathrm{d} . \mathrm{ll}}^{2 \mathrm{~b}}(R)$ for reflectivity (333) and (555).

Thus, according to the chosen model of three types of dominant defects presented in the original silicon crystals, after irradiation with high-energy electrons, a gain in reflectivity of the crystal $1 \mathrm{a}$ is caused by essential increase in diffuse component of the integral reflectivity due to enlargement of the concentration of disc-like clusters and small-size dislocation loops against reduced concentration of small spherical clusters. For the crystal $1 \mathrm{~b}$, on the contrary, reduction of its reflectivity as compared to the test sample is related to depression of diffuse component of the full integral reflectivity due to reduced concentration of dominant defects and their enlargement, whereby this sample was exposed to the highest specific radiation dose.

For the irradiated samples of series 2, negligible differences in the value of their full integral reflectivity as compared to the test sample can be caused by ordering of their defect structure due to smaller specific radiation dose and stimulation of macrodeformation field relaxation through the interdiffusion of point defects in the given samples, which resulted in improvement of their structural homogeneity.

\section{Conclusions}

From theoretical and experimental studies of silicon crystals irradiated with high-energy electrons using the method of the full integral reflectivity of X-rays it follows that:

1) $\mathrm{Cz}-\mathrm{Si}$ crystal irradiated with the dose $1.8 \mathrm{kGray}$ possesses a lager number of defects as compared to the test sample in the series which is probably related to increased concentration and size reduction of disc-like clusters and dislocation loops, which brought about an increase in full integral reflectivity value. It is observed for all the orders of reflection.

2) The above $\rho\left(\Lambda_{h k l}\right)$ dependences demonstrate typical changes in (333) and (444) reflections, which is equivalent to the thickness of the order $\Lambda_{h k l}=23 \ldots 60 \mu \mathrm{m}$, correspondingly. It may testify to the presence of regions with an increased content of microdefects (growth bands) in the bulk of the crystal.

3) Silicon crystals irradiated with double doses of high-energy electrons have lower full integral reflectivity 
as compared to the test samples due to ordering of their defect structure - reduced concentration of disc-like clusters and dislocation loops with their enlargement and the growing concentration of small-size spherical clusters.

4) Use of the method of full integral reflectivity in the crystals with some type of dominant defects as well as reflectivity dependence on the reflection order and irradiation dose allows to observe the dynamics of defectspectrum change in more details due to the possibility of quantitative estimating the contribution of each defect type into diffusion component of X-ray scattering.

\section{References}

1. T.G. Gutsulyak, V.T. Maslyuk, A.V. Oleynich-Lysyuk, N.D. Raransky, I.M. Fodchuk, Defect formation in silicon crystals after high-energy electron and $\gamma$-radiation // Ukrainsky Fizychny Zhurnal 48(9), p. 43-49 (2003) (in Ukrainian).

2. V.S. Vavilov, N.A. Ukhin, Radiation defects in semiconductors and semiconductor devices. Atomizdat Publ., Moscow, 1969 (in Russian).

3. B.N. Mukashev, Kh.A. Abdullin, Yu.V. Gorelkinsky, Metastable and bistable defects in silicon // Uspekhi Fizich. Nauk 170(2), p. 143-155 (2000) (in Russian).

4. I.I. Kolkovsky, V.V. Lukyanitsa, Peculiarities of accumulation of radiation defects of vacancy and interstitial types in dislocation-free silicon with various oxygen content // Fizika i Tekhnika Poluprovodnikov, 31(4), p. 405-408 (1997) (in Russian).

5. L.S. Berman, V.A. Zhepko, V.N. Lomasov, V.I. Tkachenko, On the nature of radiation defects in $n$-silicon exposed to electron irradiation with energy close to defect formation threshold // Ibid. 28(1), p. 2129-2131 (1989) (in Russian).

6. V.B. Neymash, M.M. Kras'ko, A.M. Kraichynsky, Generation of radiation and thermal defects in silicon under "hot" irradiation // Ukrainsky Fizychny Zhurnal, 47(1), p. 50-53 (2002) (in Ukrainian).

7. N.D. Raransky, I.M. Fodchuk, V.T. Maslyuk, A.G. Gimchynsky, P.E. Marmus, X-ray diffraction studies of structural changes in Si single crystals, irradiated with high-energy electrons // Neorganich. Mater. 33(2), p. 139-141 (1997) (in Russian).

8. V.M. Babich, N.I. Bletskan, E.F. Venger, Oxygen in silicon single crystals. Interpress Ltd, Kyiv, 1997 (in Russian).

9. O.G. Gimchinsky, T.G. Gutsulyak, A.V. OlijnichLysjuk, N.D. Raransky, I.M. Fodchuk, Evolution of defective structure of the irradiated silicon during natural ageing // Semiconductor Physics, Quantum Electronics and Optoelectronics 6(3-4), p. 43-49 (2003).
10. O.G. Gimchynsky, B.I. Gutsulyak, A.V. OleynichLysyuk, M.D. Raransky, Z. Swiatek, Structural changes of silicon crystals after high energy electron irradiation // Proc. SPIE. Bellingham. 5477, p. 215-221 (2004).

11. K. Reivi, Defects and impurities in semiconductor silicon // Mir, Moscow, 1984 (in Russian).

12. J.R. Patel, K.A. Jackson, H. Reiss, Oxygen precipitation and stacking-fault formation in dislocation-free silicon // J. Appl. Phys. 48(12), p. 5279-5288 (1977).

13. S. Mahajan, G.A. Rozgonyj, D. Brasen // Appl. Phys. Lett. 30, p. 73 (1977).

14. M.A. Krivoglaz, X-ray and neutron diffraction in nonperfect crystals. Naukova Dumka, Kyiv, 1983 (in Russian).

15. V.B. Molodkin, A.I. Nizkova, A.P. Shpak et al., Diffractometry of nanodimensional defects and crystal heterolayers. Akademperiodika, Kyiv, 2005 (in Russian).

16. A.P. Shpak, V.B. Molodkin, A.I. Nizkova, M.T. Kogut, E.V. Pervak, Influence of damaged surface layer on dynamic scattering in crystals with defects // Uspekhi Fiziki Metallov 5, p. 50-72 (2004) (in Russian).

17. V.G. Bar'yahtar, E.N. Gavrilova, V.B. Molodkin, S.I. Olikhovskii // Metallofizika 14(11), p. 68 (1992) (in Russian).

18. V.G. Bar'yahtar, M.V. Kovalchuk, Yu.M. Litvinov, et al. // Nucl. Instrum. and Meth. in Physics A, 308, p. 291 (1991) (in Russian).

19. E.N. Gavrilova, E.N. Kislovskii, V.B. Molodkin, S.I. Olikhovskii // Metallofizika 14 (3), p. 70 (1992) (in Russian).

20. V.G. Bar'yahtar, V.V. Nemoshkalenko, V.B. Molodkin et al. // Metallofizika 15 (12), p. 18 (1993) (in Russian).

21. V.V. Nemoshkalenko, V.B. Molodkin, E.N. Kislovskii et al. // Metallofiz. i Noveyshiye Tekhnologii 16 (2), p. 48 (1994) (in Russian).

22. V.B. Molodkin, S.I. Olikhivski, M.E. Osinovski et al., Integral intensity of dynamic diffraction of radiation in crystals with uniform distribution of defects // Metallofizika 6(2), p. 18-22 (1984) (in Russian).

23. I.E. Talanin, Mechanism of formation and properties of growth microdefects in dislocationfree silicon single crystals // Thesis for D. Sc. Degree. Chernivtsi, 2005.

24. V.V. Voronkov, M.G. Mil'vidsky, V.Ya. Reznik, N.I. Puzanov, A.M. Eidenson, Oxygen precipitation in silicon with various growth microdefects // Kristallografiya 35(5), p. 1197-1204 (1990) (in Russian). 